\title{
Becker Muscular Dystrophy (BMD) with Dilated Cardiomyopathy (DCM): A Case Report
}

\author{
Chen Yongliang ${ }^{1}$, Zhu Yunlong ${ }^{1}$, Zhou Yuying$^{1}$, and Jianping Zeng ${ }^{1}$ \\ ${ }^{1}$ Center of Cooperative Postgraduate Cultivation in Xiangtan Central Hospital
}

July 24, 2021

\begin{abstract}
We report a case of a BMD complicated with dilated cardiomyopathy (DCM). A 42-year-old male, his body posture and gait change began at the age of 23 years old. The patient experienced repeated shortness of breath when he was 39 years old due to dilated cardiomyopathy.
\end{abstract}

Becker Muscular Dystrophy (BMD) with Dilated Cardiomyopathy (DCM): A Case Report Yongliang Chen ${ }^{1,2, *}$, Yunlong Zhu ${ }^{2, *}$, Yuying Zhou ${ }^{1,2}$, and Jianping Zeng ${ }^{1,2,+}$

${ }^{1}$ Center of Cooperative Postgraduate Cultivation in Xiangtan Central Hospital, University of South China Xiangtan 411100, China; ${ }^{2}$ Department of Cardiology, Xiangtan Central Hospital, Xiangtan 411100, China

* Contributed equally to this work.

+Corresponding author.

Professor Dr. Jianping Zeng

Center of Cooperative Postgraduate Cultivation in Xiangtan Central Hospital

University of South China

China

Email: xhjiang2@hnust.edu.cn

Tel: +86 13973206628

Running title: Becker Muscular Dystrophy meets Dilated Cardiomyopathy Conflicts of interest

There is no conflict to be declared.

Consent

Written consent has been obtained from the patient for publication of this report.

\section{Abstract}

Background Becker muscular dystrophy (BMD) is an X-linked neuromuscular disease caused by dystrophin gene mutation, disease feature included symmetric muscle weakness and muscular atrophy. Heart could be involved in some BMD patients. 
Case Report We report a case of a BMD complicated with dilated cardiomyopathy (DCM). A 42-year-old male, his body posture and gait change began at the age of 23 years old, accompanied by weakness and muscle atrophy of both lower limbs, with a progressive aggravated trend. Fragment deletion was evidenced in exon 45-49 regions. Genetic pedigree analysis confirmed the X-linked inherited disease feature. The patient experienced repeated shortness of breath when he was 39 years old. Whole heart enlargement and aggravated cardiac dysfunction were evidenced in echocardiography examination. He was hospitalized for heart failure from time to time since then.

Conclusion BMD complicated with DCM is a rare scenario in our daily clinical practice. It is thus of importance for cardiologists to pay attention to define the primary disease course for heart failure patients with DCM. Multidisciplinary management is required in the treatment of patients with BMD complicated with cardiac damage.

Key words: Becker muscular dystrophy, X-linked recessive neuromuscular disease, dilated cardiomyopathy, heart failure.

\section{Background:}

Becker muscular dystrophy (BMD) is an X-linked neuromuscular disease caused by dystrophin gene mutation. It usually occurs in male, while females are usually the carriers of the abnormal chromosome. Patients might present with limb weakness and muscle atrophy with a chronic progressive disease course. In addition to skeletal muscle involvement, BMD can also be complicated with cardiac injury. In this case report, we presented the clinical data of a male patient with BMD complicated with dilated cardiomyopathy (DCM) and survey results of this pedigree.

\section{Case reports:}

A 42 years old male was admitted to our hospital in the year of 2019 with a complaint of 19 years history of lower limb muscle weakness and chest tightness and exertional dyspnea for 3 years. At the age of 23 , his body posture and gait began to change when climbing the stairs, accompanied by weakness and muscle atrophy of both lower limbs. Assistance by hands was required during squatting and standing up. These changes, with a progressive aggravated trend, mainly focused on the proximal muscles of both lower limbs.

Since the initiation of the disease, he repeatedly sought for medical help in various hospitals. Typical characteristics of active myogenic injure was detected by electromyogram (EMG) in 2011. Diagnosis ofDystrophinopathy was confirmed by muscle biopsy on August 2011. No special treatment was applied to him.

He developed exertional dyspnea with orthopnea at night 3 years ago. Since then, he was repeatedly hospitalized due to heart failure. Echocardiographic examination results are summarized in Table 1. 


\begin{tabular}{|c|c|c|c|c|c|c|}
\hline Date & $\begin{array}{l}\text { LAs } \\
(\mathrm{mm})\end{array}$ & $\begin{array}{l}\text { LVd } \\
(\mathrm{mm})\end{array}$ & $\begin{array}{l}\text { RAs } \\
(\mathrm{mm})\end{array}$ & $\begin{array}{l}\text { RVd } \\
(\mathrm{mm})\end{array}$ & $\begin{array}{c}\text { LVEF } \\
(\%)\end{array}$ & $\begin{array}{c}\text { PASP } \\
(\mathrm{mmHg})\end{array}$ \\
\hline 2017.01.11 & 53 & 69 & 36 & 21 & 30 & 57 \\
\hline 2017.06 .10 & 52 & 69 & 37 & 20 & 33 & 45 \\
\hline 2017.11.14 & 52 & 70 & 45 & 20 & 34 & 72 \\
\hline 2017.12.25 & 52 & 70 & 48 & 20 & 36 & 87 \\
\hline 2018.02.27 & 53 & 72 & 50 & 17 & 29 & 93 \\
\hline 2018.03 .30 & 55 & 73 & 51 & 20 & 29 & 105 \\
\hline
\end{tabular}

Table 1. Echocardiography. LAs:Left atrial end systolic diameter,LVd:Left ventricular end diastolic dimension,RAs:Right atrial end systolic diameter,RVd:Right ventricular end diastolic dimension,LVEF: left ventricular ejection fraction,PASP:pulmonary arterial systolic pressure

Genetic examinations performed in the year of 2016 revealed following heterozygous mutations: SCN5A geneC.3077>A (p.T1026k), TTN geneC.94451c > T (p.R341484Q) and TTN geneC.79693c > T (p.V265651).

Cardiac magnetic resonance (CMR) performed in the year of 2016 showed that: 1 . Left atrium $\left(81^{*} 49 \mathrm{~mm}\right)$ and left ventricle $\left(88^{*} 76 \mathrm{~mm}\right)$ were enlarged. Left ventricular systolic function was significantly decreased (LVEF: 30.3\%). 2. Left ventricular thinning was evidenced at lateral wall, middle segment of lower wall and basal segment. There was also transmural-like delayed enhancement in these segments. 3. Moderate to large mitral regurgitation. 4. A small amount of pericardial effusion.

DMD gene MLPA test was performed in 2018 and detected fragment deletion in the 45-49 region of exon of DMD gene was evidenced.

Right heart catheterization was performed in the year of 2018 with following results: Pulmonary arterial systolic pressure $=87 \mathrm{mmHg}$,pulmonary capillary wedge pressure $=29 \mathrm{mmHg}$, the difference value of diastolic pulmonary pressure $=28 \mathrm{mmHg}$, total pulmonary resistance $=16.75 \mathrm{wood}$, pulmonary vascular resistance $=8.38$ wood, $Q p /$ Qs ratio $=1.02$.

Genetic pedigree analysis was finished in 2018, results confirmed that the disease conformed to the recessive inheritance characteristic of X-chromosome (Table 2). Despite presence of related heterogeneous gene mutations in some family members, none of his family members was clinically ill, and no symptoms and signs of Becker muscular dystrophy or DCM were found in his family members.

Table 2. Gene analysis for the family members 


\section{Gene family analysis}

\begin{tabular}{|c|c|c|c|}
\hline Gene & $\begin{array}{c}\text { SCN5A } \\
\text { c.3077C>A(p.T1 } \\
\text { 026k) }\end{array}$ & $\begin{array}{c}\text { TTN } \\
\text { c.94451C>T(p.R31484 } \\
\text { Q) }\end{array}$ & $\begin{array}{c}\text { TTN } \\
\text { c.79693C> T(p.V26565 } \\
\text { 1) }\end{array}$ \\
\hline patiet & + & + & + \\
\hline father & + & - & + \\
\hline mother & - & + & - \\
\hline daughter & + & + & - \\
\hline $\begin{array}{c}\text { elder } \\
\text { brother }\end{array}$ & + & - & + \\
\hline $\begin{array}{c}\text { second } \\
\text { elder } \\
\text { brother }\end{array}$ & + & + & \\
\hline
\end{tabular}

\section{Clinical presentation}

Blood pressure was $86 / 60 \mathrm{mmHg}$, heart rate was 90 beats per minute at admission. Patient was sanity, tachypnea with engorgement of the neck veins. Low breath sounds in his both lungs and soft rales could be heard in the base of both lungs. Abdomen was flat and soft, there was no tenderness and rebound tenderness. Edema was found in both lower limbs. Meanwhile, there were signs of symmetrical atrophy thigh muscles, hypertrophy and stiffness gastrocnemius, and the limbs skin temperature was low. Laboratory examination results were as follows: Creatine Kinase (CK): 566IU/L, Myohemoglobin: 219.2ug/L, Urea nitrogen: 8.5mmol/L,Hemoglobin: 94g/L, Neutrophilic granulocyte percentage (NEUT):76.2\%, Troponin I (TnI): $<0.5 \mathrm{ng} / \mathrm{ml}$. BNP:4700pg/ml, NT-proBNP:5732 pg/ml. Electrocardiograph (ECG) results were as follows: sinus rhythm. Ventricular premature beat (Figure 1). CT scan of the lungs showed signs of inflammatory response in lung field, small effusion in bilateral pleural cavity. Echocardiography detected enlarged left ventricle (LVEDD $78 \mathrm{~mm}$ ), left atria $(\mathrm{LADs}=55 \mathrm{~mm})$, right atria $($ RAs $54 \mathrm{~mm})$ and right ventricle $(\mathrm{RVd}$ $37 \mathrm{~mm})$ as well as global hyperkinesia $(\mathrm{LVEF}=20 \%)$, moderate to large mitral regurgitation and significant tricuspid regurgitation (systolic pulmonary artery pressure $=72 \mathrm{mmHg}$ ).

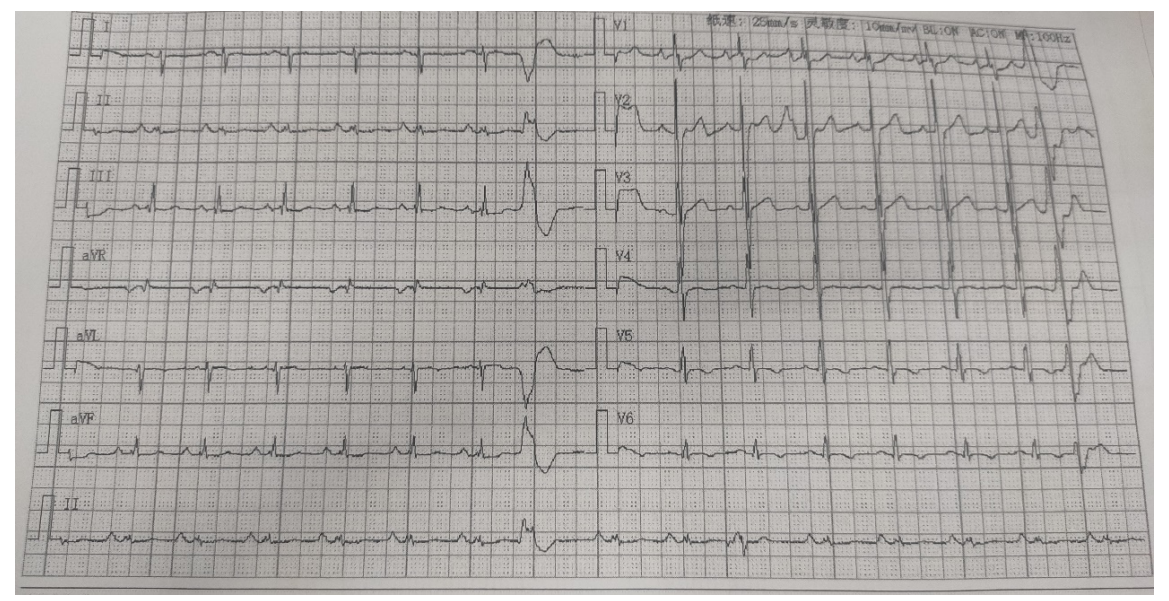

Figure 1.

\section{Therapy:}


After admission, he was treated with methylprednisolone (4mg/day), torasemide (10mg/day), sacubitril/valsartan $(2 \times 25 \mathrm{mg} /$ day $)$, bosentan $(2 \times 125 \mathrm{mg} /$ day $)$, Ivabradine $(2 \times 7.5 \mathrm{mg} /$ day $)$, benzbromaron $(50 \mathrm{mg} /$ day $)$, rosuvastatin $(10 \mathrm{mg} /$ day $)$. The symptoms were improved and patients were discharged after 9 days hospitalization and he was recommended to keep on above medication regimen after discharge and will be followed up regularly in our outpatient unit.

\section{Discussion:}

Becker muscular dystrophy is one of the two common forms of Duchenne muscular dystrophy, which is a $\mathrm{X}$-linked recessive genetic disease. It usually occurs in male, while female are the carriers of the abnormal chromosome.

According to the difference of onset age and disease course, patients with dystrophinopathy are divided into Duchenne's muscular dystrophy (DMD) and Becker's muscular dystrophy (BMD), both of which are caused by the encoding gene absence and partially-absence of dystrophin (1).

The involved gene is located in the XP21.2 region of sex chromosome, and is the largest gene known to human at present. It is about $2.4 \mathrm{Mb}$ in length, contains 79 exons and 79 introns, and encodes mRNA about $14 \mathrm{~KB}$. The translated product is anti-dystrophin, which is located in the lipid of muscle cell membrane, and which plays an important role in stabilizing cell membrane and preventing cell necrosis and autolysis. The main types of gene absence include exon deletion, duplication and gene micromutation $(2,3)$.

The main manifestations of Duchenne muscular dystrophy (DMD/BMD) in the early stage are atrophy and weakness of the proximal lower extremities muscular and pelvic girdle, pseudohypertrophy of calf gastrocnemius, duck step and Gowers sign. In the late disease stage, systemic skeletal muscle atrophy, myocardial involvement, featured by myocardial fibrosis and adipose tissue infiltration might occur. The myocardial involvement is usually resulted from the absence of myocardial dystrophy protein. The atrophy degeneration, fibrosis and infiltration of adipose tissue of the left ventricular myocardium could lead to abnormal ventricular wall motion, and eventually dilated cardiomyopathy and severe arrhythmias.

Clinically, disease course of DMD patients is progressive and the prognosis is poor. About $1 / 3$ of the patients might suffer from mental retardation and usually die of respiratory or heart failure in their 20s. BMD is an allelic disease, which is relatively rare and patients usually present with the general characteristics of Duchenne muscular dystrophy. However, the disease progress of BMD is relatively slow, the average age of disease onset is late and survival outcome is better than DMD, cognitive impairment is usually absent in BMD patients.

In our case, disease onset of the disease is late, clinical manifestations are typical for Duchenne muscular dystrophy, combined with typical changes on serum enzymology, electromyography, pathology and genetics. He was first diagnosed as "pseudo hypertrophic muscular dystrophy". Then, based on the late onset and slow progression disease feature and absence of cognitive impairment, patient was diagnosed as Becker muscular dystrophy (BMD). In addition, echocardiography examination indicated whole heart enlargement with reduced LVEF, and CMR indicated myogenic injury, so the diagnosis of DCM was established, which might be secondary to BMD.

The most common cause of death in BMD is DCM and associated heart failure (4). The patient reported here did suffer from severe heart failure and our patient presented typical signs and symptoms of DCM-related heart failure with NYHA classification III-IV. It is of importance to define the disease feature and cardiac structural and function changes with the help of echocardiography, magnetic resonance imaging and other methods in BMD patients with or without symptoms of heart failure and monitor related changes thereafter (5).

At present, there is no effective radical treatment for Duchenne muscular dystrophy . Genetic counseling and prenatal diagnosis for high-risk pregnant women with related family history are recommended in some countries. Multidisciplinary management of DMD/BMD patients is of importance aiming to slow down disease progression, and improve quality of life and outcome. Glucocorticoid is the only proved drug by 
evidence-based medicine to delay the clinical course of DMD (6), which can delay muscle strength decline, muscle contracture, joint deformation and heart damage. Clinicians should pay attention to potential adverse reactions related long-term glucocorticoid treatment in these patients. For BMD patients combined with cardiac damage and without heart failure symptoms, angiotensin converting enzyme inhibitors (ACEI) and $\beta$-blockers could delay progression of left ventricular dysfunction (7). Sodium-glucose transport protein 2 (SGLT2) inhibitors are new-comers for HF patients with preserved or reduced LVEF (ref), efforts should be made to observe if BMD patients with heart failure could benefit from this medication or not. Left ventricular assist device shows a certain application prospect in improving quality of life and survival in BMD patients (8). In addition, impact of other treatment methods, such as gene therapy, cell transplantation and heart transplantation therapy, is under exploration now.

\section{Conclusion:}

This report describes a case of BMD complicated with dilated cardiomyopathy, which is a rare case in our daily clinical practice. It is thus of importance for cardiologists to pay attention to define the primary disease etiology for heart failure patients with dilated cardiomyopathy. Multidisciplinary management is required in the treatment of patients with BMD complicated with cardiac injury.

Acknowledgements: supported by grant from scientific bureau of Xiangtan city, Hunan province, China (SF-YB20201023).

\section{References:}

1. Flanigan KM. Duchenne and Becker muscular dystrophies. Neurologic clinics. 2014;32(3):671-88, viii.

2. Aartsma-Rus A, Van Deutekom JC, Fokkema IF, Van Ommen GJ, Den Dunnen JT. Entries in the Leiden Duchenne muscular dystrophy mutation database: an overview of mutation types and paradoxical cases that confirm the reading-frame rule. Muscle \& nerve. 2006;34(2):135-44.

3. Takeshima Y, Yagi M, Okizuka Y, Awano H, Zhang Z, Yamauchi Y, et al. Mutation spectrum of the dystrophin gene in 442 Duchenne/Becker muscular dystrophy cases from one Japanese referral center. Journal of human genetics. 2010;55(6):379-88.

4. Hoogerwaard EM, de Voogt WG, Wilde AA, van der Wouw PA, Bakker E, van Ommen GJ, et al. Evolution of cardiac abnormalities in Becker muscular dystrophy over a 13-year period. Journal of neurology. 1997;244(10):657-63.

5. de Souza F, Bittar Braune C, Dos Santos Nucera APC. Duchenne muscular dystrophy: an overview to the cardiologist. Expert review of cardiovascular therapy. 2020;18(12):867-72.

6. Escolar DM, Hache LP, Clemens PR, Cnaan A, McDonald CM, Viswanathan V, et al. Randomized, blinded trial of weekend vs daily prednisone in Duchenne muscular dystrophy. Neurology. 2011;77(5):44452 .

7. Chamberlain JS. ACE inhibitor bulks up muscle. Nature medicine. 2007;13(2):125-6.

8. Hayes EA, Nandi D. Is there a future for the use of left ventricular assist devices in Duchenne muscular dystrophy? Pediatric pulmonology. 2021;56(4):753-9. 